\title{
BMJ Open Response to cardiac resynchronisation therapy in men and women: a secondary analysis of the SMART-AV randomised controlled trial
}

\author{
Stacey Howell, ${ }^{1}$ Timothy M Stivland (D , ${ }^{2}$ Kenneth Stein, ${ }^{2}$ Kenneth Ellenbogen, ${ }^{3}$ \\ Larisa G Tereshchenko (iD ${ }^{1,4}$
}

To cite: Howell S, Stivland TM, Stein K, et al. Response to cardiac resynchronisation therapy in men and women: a secondary analysis of the SMART-AV randomised controlled trial. BMJ Open 2021;11:e049017. doi:10.1136/ bmjopen-2021-049017

- Prepublication history and additional supplemental material for this paper are available online. To view these files, please visit the journal online (http://dx.doi.org/10.1136/ bmjopen-2021-049017).

Received 14 January 2021 Accepted 07 October 2021

Check for updates

(C) Author(s) (or their employer(s)) 2021. Re-use permitted under CC BY-NC. No commercial re-use. See rights and permissions. Published by BMJ.

${ }^{1}$ Department of Medicine, Oregon Health \& Science University School of Medicine, Portland, Oregon, USA

${ }^{2}$ Boston Scientific Corp, Marlborough, Massachusetts, USA

${ }^{3}$ Department of Medicine, Medical College of Virginia, Richmond, Virginia, USA

${ }^{4}$ Department of Medicine, Johns Hopkins University School of Medicine, Baltimore, Maryland, USA

Correspondence to Dr Larisa G Tereshchenko; tereshch@ohsu.edu

\section{ABSTRACT}

Objectives There is a controversy about whether both sexes' response to cardiac resynchronisation therapy (CRT) is similar. We aimed to assess a causal effect of sex on CRT response.

Design Secondary analysis of a randomised controlled trial (RCT) data. Doubly robust augmented-inverseprobability-weighted (AIPW) estimation of sex effect on CRT response.

Setting The SmartDelay Determined Atrioventricular (AV) Optimisation (SMART-AV) RCT.

Participants The SMART-AV RCT enrolled New York Heart Association class III-IV patients with heart failure (HF) with left ventricular ejection fraction (LVEF) $\leq 35 \%$ despite optimal medical therapy and QRS duration $\geq 120$ $\mathrm{ms}$, in sinus rhythm. After exclusion of those with missing outcome or covariates, 741 participants (age $66 \pm 11$ years; $33 \%$ female; $78 \%$ white; LVEF $28 \% \pm 9 \%$; $58 \%$ ischaemic cardiomyopathy; $75 \%$ left bundle branch block; left ventricular end-systolic volume index (LVESVI) $65 \pm 30 \mathrm{~mL} /$ $\mathrm{m}^{2}$ ) were included.

Interventions Implanted CRT defibrillator with randomly assigned AV delay as either (1) fixed at $120 \mathrm{~ms}$, or (2) echocardiography-determined, or (3) SmartDelay algorithm-programmed.

Outcome A composite of freedom from death and HF hospitalisation and a $>15 \%$ reduction in LVESVI at 6 month post-CRT was the endpoint.

Results The primary endpoint was met by 337 patients (45.5\%); 134 were women (55.6\% response) and 203 were men ( $40.6 \%$ response); $p<0.0001$. After conditioning for 33 covariates that included baseline demographic, clinical, ECG, echocardiographic and biomarker characteristics, known predictors of CRT response, logistic regression showed a higher probability for composite CRT response for women versus men (OR 1.79; 95\% $\mathrm{Cl} 1.08$ to 2.98; $p<0.0001$ ), whereas AIPW estimation showed no difference in CRT response (average treatment effect 0.88 ; $95 \% \mathrm{Cl} 0.41$ to 1.89; $\mathrm{p}=0.739$ ). After removing colliders from the model, both logistic regression (OR 1.00; $95 \% \mathrm{Cl}$ 0.69 to 1.44 ) and AIPW (ATE $1.06 ; 95 \% \mathrm{Cl} 0.96$ to 1.16) reported similar results

Conclusions Both sexes' response to CRT is similar. Sex differences in HF substrate, treatment and comorbidities explain sex disparities in CRT outcomes.
Strengths and limitations of this study

- SmartDelay Determined Atrioventricular (SMARTAV) optimisation was a large multicentre randomised control trial that included blinded analysis of echocardiograms in core laboratory and diligent follow-up.

- While the echocardiographic SMART-AV core laboratory was blinded to the randomisation assignment, it was not blinded to participants' sex and echocardiogram type (pre-or post-cardiac resynchronisation therapy (CRT)).

- The study population was predominantly composed of men. The heterogeneity of CRT response by sex was not prespecified SMART-AV analysis.

- The present study's outcome differed from the prespecified original SMART-AV randomised controlled trial primary endpoint (change in left ventricular end-systolic volume index (LVESVI) at 6 months post-CRT)

- The current secondary SMART-AV analysis used a composite endpoint of clinical outcomes (death, HF hospitalisation) and volumetric remodelling (>15\% reduction in LVESVI at 6-month post-CRT), and, therefore, reduced attrition bias.

Trial registration number ClinicalTrials.gov Identifier; NCT00677014.

\section{INTRODUCTION}

Cardiac resynchronisation therapy (CRT) is an established treatment for patients with systolic heart failure (HF) and ventricular dyssynchrony. ${ }^{1} \quad$ However, despite proven benefits, nearly a-third of CRT recipients are considered to be 'non-responders. ${ }^{, 2}$ Furthermore, although many previous studies have suggested that the female sex is associated with a higher responder rate, ${ }^{34}$ there is still controversy: some studies determined that the response of both sexes to CRT is similar. ${ }^{5-7}$

Guided left ventricular (LV) lead placement considering the timing of LV activation 
and electrical delay, ${ }^{8}$ together with dynamic atrioventricular (AV) optimisation, ${ }^{9}$ can potentially reduce the CRT non-response rate. Previous analysis of the SmartDelay determined AV Optimisation: (a comparison to other AV delay methods used in CRT) study showed an enhanced response to $\mathrm{AV}$ optimisation in women as compared with men. ${ }^{10}$

Notably, previous research on sex differences in CRT response did not evaluate the causal effect of sex, which requires the counterfactual analytical framework. Conducting causal inference studies about sex is necessary to mitigate inequalities, reduce disparities, and, if needed, facilitate sex-specific treatment interventions. To address this knowledge gap, we conducted the current study to determine the causal effect of sex on CRT response.

\section{METHODS}

\section{Study population}

The SMART-AV was a randomised, multicentre, singleblinded clinical trial $^{11} 12$ that sought to determine whether AV delay optimisation would improve CRT response 6-month postimplant. The trial enrolled New York Heart Association (NYHA) class III-IV patients with HF with left ventricular ejection fraction (LVEF) $\leq 35 \%$ despite optimal medical therapy, and QRS duration $\geq 120$ ms, in sinus rhythm. Patients with HF in complete heart block could not tolerate pacing at VVI-40-RV for up to 2 weeks, or previously received CRTs were excluded. Enrolment was completed from May 2008 through December 2009. In the current study, we excluded participants with missing baseline clinical characteristics data and lost to follow-up (figure 1). Of the 980 randomised SMART-AV participants, 741 CRT recipients were included in this study.

\section{Baseline clinical characteristics}

At the enrolment visit, baseline clinical characteristics data were collected, which included medical history, current cardiovascular evaluation (NYHA class) and medications list. ${ }^{1112}$ We calculated the estimated glomerular filtration rate (eGFR) using the chronic kidney disease (CKD) Epidemiology Collaboration equation $\quad$ (CKD-EPI) ${ }^{13}$ Baseline ECG and echocardiogram were recorded postimplant (no biventricular pacing). ${ }^{11} 12$ We normalised LV volumes and dimensions by body surface area (BSA).

\section{The study endpoint}

In the current study, we defined the primary endpoint as a composite of freedom from death and HF hospitalisation and $\mathrm{a}>15 \%$ reduction $^{9} 101415$ in LV end-systolic volume index (LVESVI) at 6 months of follow-up. LVESV was the primary endpoint in the SMART-AV trial. ${ }^{11}{ }^{12} \mathrm{~A}$ single core laboratory performed all echocardiographic measurements in a blinded fashion.

\section{Statistical analysis}

Normally distributed continuous variables were compared using the t-test and reported as mean $\pm \mathrm{SD}$. Categorical

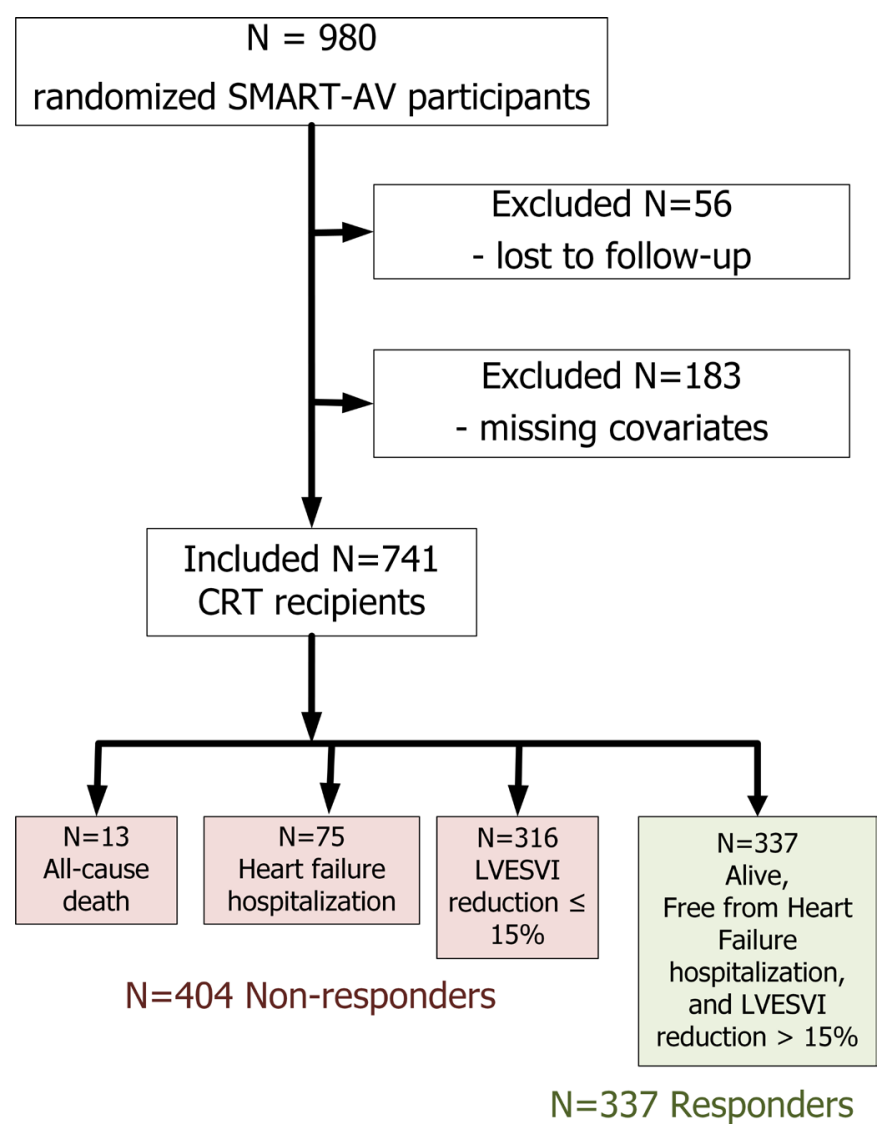

Figure 1 Flowchart of study cohort development. AV, atrioventricular; CRT, cardiac resynchronisation therapy; LVESVI, left ventricular end-systolic volume index.

variables were compared using the $\chi^{2}$ test. Univariate logistic regression compared the odds of CRT response.

To assess the effect of sex on CRT response, we used augmented-inverse-probability-weighted (AIPW) estimators, ${ }^{16}$ as implemented in STATA (teffects aipw). AIPW estimators have the double-robust property. AIPW estimators model both the outcome (CRT response) probability and the probability of being man or woman (using the counterfactual framework). Both logistic models were conditioned on the baseline covariates. We constructed three AIPW models. Model 1 was conditioned only for BSA and body mass index (BMI). Model 2 was conditioned for 33 covariates: demographic (age, race) and body type characteristics (BMI, BSA), cardiac disease substrate (cardiomyopathy type, ventricular conduction abnormality type, NYHA HF class, AV block, revascularisation history, cardiac valve disease, QRS duration, PR interval, heart rate, LVEF, LV end-diastolic volume index (LVEDVI), LVESVI, LV end-diastolic dimension index (LVEDDI), LV end-systolic dimension index, risk factors and comorbidities (hypertension, systolic and diastolic blood pressure, diabetes, smoking, autoimmune disease, cancer, chronic obstructive pulmonary disease, sleep apnea,

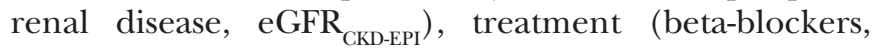
angiotensin-converting enzyme inhibitors, angiotensin receptor blockers (ARBs), aldosterone antagonists) and defibrillator indication (primary or secondary prevention 
of sudden cardiac death). The Imai and Ratkovic ${ }^{17}$ test was used to verify the covariates balance, illustrated by overlap plots for densities of predicted probabilities for men and women.

It is known that if a regression is conditioned on a collider, it is prone to selection bias. ${ }^{18}$ Conditioning on colliders, time-dependent confounding and mediation can produce paradoxical effects in regression analysis. Causal inference approach using doubly robust estimators was developed to avoid the limitations of regression analysis. Therefore, we compared the average treatment effect (ATE) reported by AIPW estimators with logistic regression OR. The ATE is interpreted as the population risk difference in 6-month CRT response for women compared with men. We constructed multivariable logistic regression models 1 and 2 with the same covariate list as AIPW models. In addition, we classified all covariates included in model two into 'confounder' or 'collider' categories, using an iterative Leave-One-Out approach (online supplemental table 1). Covariates that attenuated the association (ie, their presence in the model decreased point estimate) between sex and CRT response were confounders. Covariates that strengthened the association (ie, their presence in the model increased point estimate) between sex and CRT response were colliders. Then, we constructed multivariable logistic regression and AIPW models 3, including confounders and excluding all colliders.

To investigate whether the definition of the primary endpoint affected the study results, we conducted sensitivity analysis after excluding study participants who missed 6 month post-CRT echocardiographic assessment because of death or HF hospitalisation. A decrease in LVESVI $>15 \% 6$ month post-CRT was used as the endpoint for sensitivity analysis.

Statistical analysis was performed using STATA MP V.16.1 (StataCorp LP, College Station, Texas). P value $<0.05$ was considered statistically significant. STATA code is provided at https://githubcom/Tereshchenkolab/ statistics.

\section{Patient and public involvement}

Patients or the public were not involved in the design, conduct, reporting of this study or dissemination of results.

\section{RESULTS}

\section{Study population}

The SMART-AV study population characteristics in men and women were vastly different, as shown in table 1 , and have been previously reported elsewhere. ${ }^{10}$ At baseline, men were more likely white, with ischaemic cardiomyopathy, AV block, had more comorbidities, a lower LVEF and higher LVESVI and LVEDVI. Overall, men were more likely to have ventricular conduction abnormality types known to be less likely to respond to CRT.

\section{Comparison of the response of men and women to CRT}

The primary endpoint was met by 337 patients (45.5\%), 134 were women (55.6\% response) and 203 were men ( $40.6 \%$ response); $\mathrm{p}<0.0001$. Out of 404 participants who failed to respond, 13 died (10 men and 3 women), 75 participants (53 men and 22 women) were hospitalised because of HF, and 316 (334 men and 82 women) participants failed to achieve a volumetric response (figure 2).

Unadjusted logistic regression analysis showed that women had an $80 \%$ higher probability for composite CRT response than men (OR 1.83; 95\% CI 1.34 to 2.50); $\mathrm{p}<0.0001$ ); see figure 3. Adjustment for BMI and BSA in logistic regression model one indicated even higher odds of composite CRT response for women than men, by more than twofold. After adjustment for all 33 covariates in logistic regression model 2, OR was similar to that in the unadjusted model. Further analysis of covariates showed that age, race, BMI, BSA, LVEF and all LV volume and dimension indices, smoking, history of diabetes and autoimmune disease, eGFR, heart rate, QRS duration and use of aldosterone antagonists strengthened the association of sex with CRT response and were classified as 'colliders (figure 4). 'Strengthened the association' meant that their presence in the model resulted in greater value of point estimate for sex (online supplemental table 1). Adjustment for confounders only in model 3 revealed that there was no statistically significant difference in the odds of composite CRT response between men and women, with an OR of exactly 1.00 (figure 3 ).

AIPW estimation showed no significant difference in CRT response for women compared with men, both in model 1 (ATE $1.05 ; 95 \%$ CI 0.58 to $1.91 ; \mathrm{p}=0.875$ ) and model 2 (ATE 0.88 ; 95\% CI 0.41 to 1.89 ; $\mathrm{p}=0.739$ ). Covariates were satisfactory-balanced in model $2\left(\chi^{2}(39)=18.1\right.$; $\mathrm{p}=0.998$ ), but not in model 1 (figure 5). Conditioning for confounders only in model 3 resulted in satisfactorybalanced covariates and dramatic shrinking of CIs (figure 5). Notably, ATE estimates in all three AIPW models were consistently close to 1 , indicating no difference in 6-month CRT response for women compared with men.

\section{Sensitivity analysis}

After the exclusion of 31 participants, 710 were included in the sensitivity analysis. Volumetric CRT response was observed in 351 individuals $(49.4 \% ; 210$ men and 141 women), whereas 359 (50.6\%; 267 men and 92 women) were non-responders. The results of logistic regression and AIPW models with volumetric response were nearly identical to the main study results (table 2).

\section{DISCUSSION}

In this large study, using the counterfactual framework with doubly robust estimators, we showed that there is no significant effect of sex on the probability of CRT response. Frequently observed better CRT outcomes in women than men are explained by the sex differences 
Table 1 Baseline clinical characteristics in men and women

\begin{tabular}{|c|c|c|c|c|}
\hline Characteristics & All $(n=741)$ & Women $(n=241)$ & Men $(n=500)$ & $P$ value \\
\hline Age (SD), years & $66.0(11.0)$ & $64.8(11.4)$ & $66.5(10.7)$ & 0.046 \\
\hline White, n (\%) & $575(77.6)$ & $172(71.4)$ & $403(80.6)$ & 0.005 \\
\hline LVEF (SD), \% & $27.5(8.7)$ & $28.5(8.7)$ & $27.0(8.7)$ & 0.031 \\
\hline Weight (SD), kg & $87.4(20.8)$ & $79.2(19.0)$ & $91.3(20.4)$ & $<0.0001$ \\
\hline Height (SD), cm & $171.6(10.3)$ & $161.9(7.7)$ & $176.3(7.7)$ & $<0.0001$ \\
\hline Body mass index (SD), kg/m² & $29.6(6.2)$ & $30.2(6.7)$ & $29.3(6.0)$ & 0.086 \\
\hline Body surface area (SD), $\mathrm{m}^{2}$ & $2.03(0.27)$ & $1.88(0.24)$ & $2.10(0.25)$ & $<0.0001$ \\
\hline BP systolic (SD), mm Hg & $124.5(20.9)$ & $125.9(23.1)$ & $123.9(19.8)$ & 0.246 \\
\hline BP diastolic (SD), mm Hg & $71.4(12.7)$ & $69.9(12.9)$ & $72.0(12.6)$ & 0.036 \\
\hline Ischaemic cardiomyopathy Hx, n (\%) & $426(57.5)$ & $90(37.3)$ & $336(67.2)$ & $<0.0001$ \\
\hline Primary prevention, $\mathrm{n}(\%)$ & $589(79.5)$ & $211(87.6)$ & $378(75.6)$ & $<0.0001$ \\
\hline Smoking $\mathrm{Hx}$ (current or former), $\mathrm{n}(\%)$ & $461(62.2)$ & $113(46.9)$ & $348(72.6)$ & $<0.0001$ \\
\hline Hypertension Hx, n (\%) & $528(71.3)$ & $173(71.8)$ & $355(71.0)$ & 0.220 \\
\hline Diabetes Hx, n (\%) & $289(39.0)$ & $93(38.6)$ & $196(39.2)$ & 0.873 \\
\hline Revascularisation $\mathrm{Hx}, \mathrm{n}(\%)$ & $380(51.3)$ & $76(31.5)$ & $304(60.8)$ & $<0.0001$ \\
\hline Autoimmune disease $\mathrm{Hx}, \mathrm{n}(\%)$ & $19.0(2.6)$ & $9(3.7)$ & $10(2.0)$ & 0.162 \\
\hline Sleep apnea Hx, n (\%) & $89(12.0)$ & $18(7.5)$ & $71(14.2)$ & 0.008 \\
\hline Cancer Hx, n (\%) & $67(9.0)$ & $24(10.0)$ & $43(8.6)$ & 0.546 \\
\hline Renal disease $\mathrm{Hx}, \mathrm{n}(\%)$ & $119(16.1)$ & $25(10.4)$ & $94(18.8)$ & 0.003 \\
\hline COPD Hx, n (\%) & $109(14.7)$ & $25(10.4)$ & $84(16.8)$ & 0.021 \\
\hline Valve disease $\mathrm{Hx}, \mathrm{n}(\%)$ & $40(5.4)$ & $12(5.0)$ & $28(5.6)$ & 0.726 \\
\hline AV block, n (\%) & $138(18.6)$ & $19(7.9)$ & $119(23.8)$ & $<0.0001$ \\
\hline PR interval (SD), ms & $198.2(50.4)$ & $183.2(36.7)$ & $205.4(54.6)$ & $<0.0001$ \\
\hline Heart rate (SD), bpm & $71.3(12.5)$ & $72.8(12.7)$ & $70.5(12.4)$ & 0.021 \\
\hline QRS duration (SD), ms & $151.8(19.9)$ & $151.3(17.1)$ & $152.0(21.1)$ & 0.6410 \\
\hline Conduction disease: LBBB, n (\%) & $552(74.5)$ & $204(84.7)$ & $348(69.6)$ & $<0.0001$ \\
\hline RBBB & $81(10.9)$ & $13(5.4)$ & $68(13.6)$ & \\
\hline IVCD & $86(11.6)$ & $18(7.5)$ & $68(13.6)$ & \\
\hline RBBB +left hemiblock & $22(3.0)$ & $6(2.5)$ & $16(3.2)$ & \\
\hline NYHA class II, n (\%) & $21(2.8)$ & $5(2.1)$ & $16(3.2)$ & 0.588 \\
\hline III & $698(94.2)$ & $230(95.4)$ & $468(93.6)$ & \\
\hline IV & $22(3.0)$ & $6(2.5)$ & $16(3.2)$ & \\
\hline Potassium(SD), mmol/L & $4.3(0.5)$ & $4.2(0.5)$ & $4.3(0.5)$ & 0.001 \\
\hline Sodium(SD), $\mathrm{mmol} / \mathrm{L}$ & $138.7(3.1)$ & $138.9(3.1)$ & $138.6(3.2)$ & 0.224 \\
\hline $\mathrm{eGFR}_{\mathrm{CKD}-\mathrm{EPI}}(\mathrm{SD}), \mathrm{mL} / \mathrm{min} / 1.73 \mathrm{~m}^{2}$ & $63.6(22.8)$ & $64.5(22.6)$ & $63.2(22.9)$ & 0.484 \\
\hline Use of ACEI/ARB, $n(\%)$ & $485(65.5)$ & $156(64.7)$ & $329(65.8)$ & 0.774 \\
\hline Use of beta blocker, n (\%) & $681(91.9)$ & $223(92.5)$ & $458(91.6)$ & 0.663 \\
\hline Use of aldosterone antagonist, $\mathrm{n}(\%)$ & $262(35.4)$ & $99(41.1)$ & $163(32.6)$ & 0.024 \\
\hline LV end systolic volume index (SD), mL/m² & $64.7(29.8)$ & $58.1(25.2)$ & $68.0(31.0)$ & $<0.0001$ \\
\hline LV end diastolic volume index (SD), $\mathrm{mL} / \mathrm{m}^{2}$ & $87.0(32.0)$ & $79.3(27.3)$ & $90.7(33.5)$ & $<0.0001$ \\
\hline LV end systolic diameter index (SD), cm/ $\mathrm{m}^{2}$ & $2.8(0.5)$ & $2.8(0.5)$ & $2.7(0.5)$ & 0.031 \\
\hline LV end diastolic diameter index (SD), $\mathrm{cm} / \mathrm{m}^{2}$ & $3.2(0.5)$ & $3.2(0.5)$ & $3.1(0.5)$ & 0.004 \\
\hline
\end{tabular}

$\mathrm{ACEl}$, angiotensin-converting enzyme inhibitor; ARB, angiotensin receptor blocker; CKD-EPI, chronic kidney disease epidemiology collaboration equation; eGFR, estimated glomerular filtration rate; LBBB, Left bundle branch block; LV, left ventricular; NYHA, New York Heart Association; RBBB, Right bundle branch block. 
All participants
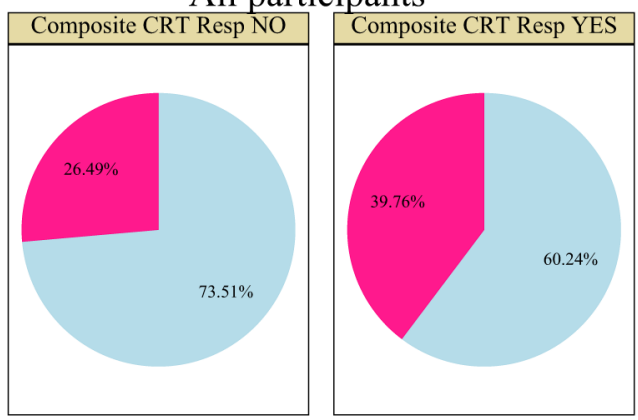

Composite Non-Responders
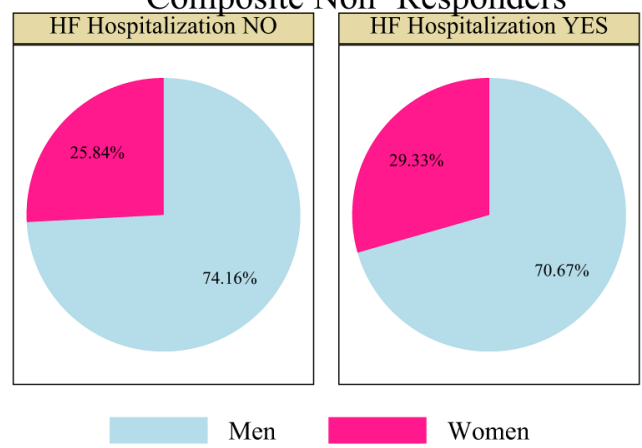

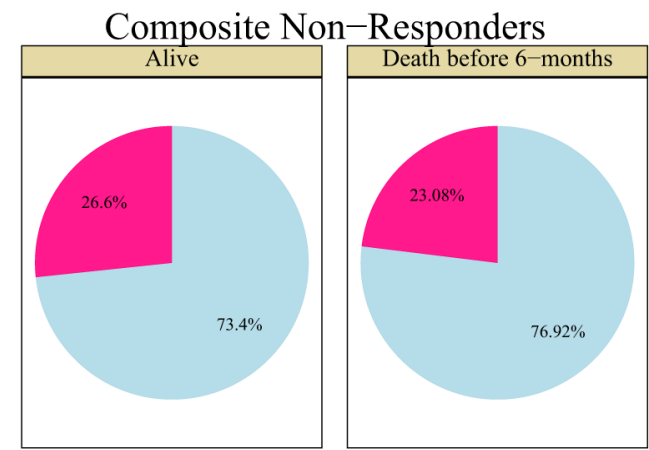

Composite Non-Responders

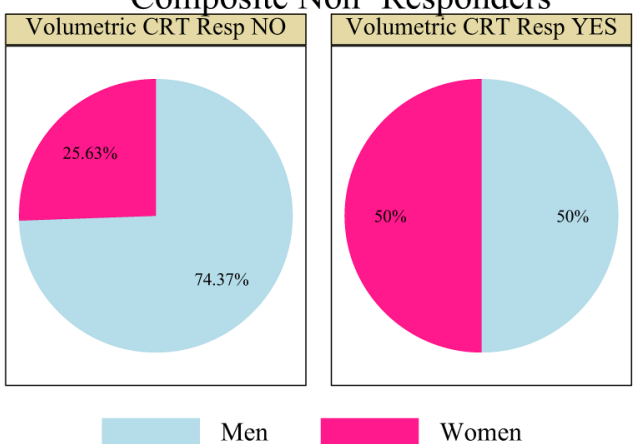

Figure 2 Proportion of men and women among composite CRT responders and non-responders for all participants, and for subgroups of composite CRT non-responders. CRT, cardiac resynchronisation therapy; HF, heart failure.

in baseline demographic and clinical characteristics, including the disease substrate, dyssynchrony, comorbidities and HF treatment. This study illustrated the impact of paradoxical effects in regression analysis arising from conditioning on colliders or effect modifiers. 18

$$
\begin{aligned}
& 1.06(0.96-1.16) \\
& 0.88(0.41-1.89) \\
& 1.05(0.58-1.91)
\end{aligned}
$$$$
1.00(0.69-1.44)
$$$$
1.79(1.08-2.98)
$$$$
2.28(1.50-3.46)
$$$$
1.83(1.34-2.50)
$$

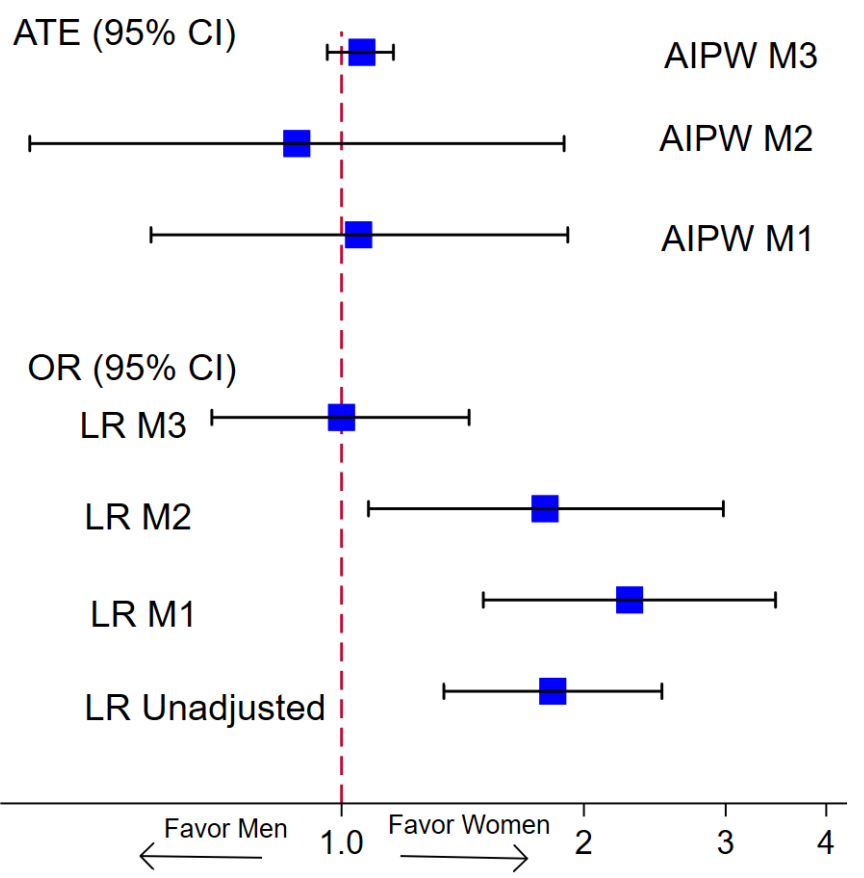

Odds of CRT response for women versus men

Figure 3 Relative odds of the composite cardiac resynchronisation therapy (CRT) response for women compared with men, by logistic regression (LR), and augmented-inverse-probability-weighted (AIPW) average treatment effect (ATE) estimator in models 1-3. The forest plot shows the relative OR or ATE OR with a $95 \% \mathrm{Cl}$ for women compared with men, with OR/ATE=1 for men. Black lines correspond to $95 \% \mathrm{Cl}$ bounds. Model 1 was conditioned for body mass index and body surface area. Model 2 was conditioned on 33 covariates, and model 3 was conditioned on confounders only. 


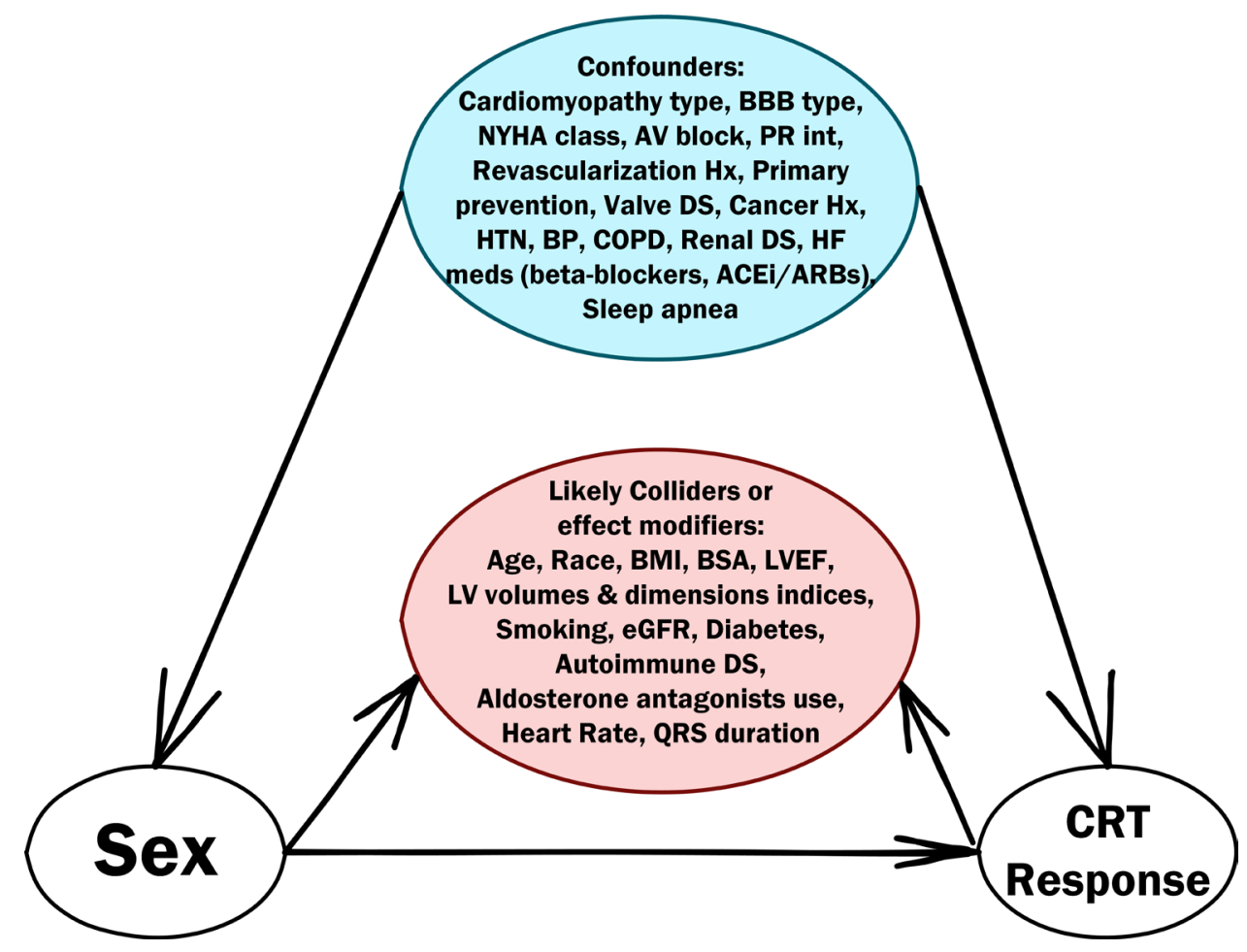

Figure 4 Direct acyclic graph. The average treatment effect is interpreted as the population risk difference in 6 month CRT response for women compared with men. ACEI, angiotensin-converting enzyme inhibitor; ARB angiotensin receptor blocker; AV, atrioventricular; BP, blood pressure; BMI body mass index; BSA, body surface area; COPD, chronic obstructive pulmonary disease; CRT, cardiac resynchronisation therapy; eGFR, estimated glomerular filtration rate; HF, heart failure; LVEF, left ventricular ejection fraction; NYHA, New York Heart Association; BBB, bundle branch block; DS, disease; Hx, history; HTN, hypertension.

Previous regression analysis of the SMART-AV data ${ }^{10}$ adjusted only for key sex differences in baseline characteristics, which included both confounders and colliders. Previous regression analyses comparing CRT outcomes in men and women faced limitations due to the relatively small proportion of female participants and, therefore, could not conduct comprehensive adjustment for all known sex differences. To compensate for the noticeable difference in sex subgroup fractions and baseline characteristics of men and women in the study, ${ }^{10}$ we used a counterfactual analytical framework and conditioned for 33 covariates. To ensure that we do not overfit our model, we constructed two AIPW models: model 1 was conditioned only for BMI and BSA. Nevertheless, both models showed very similar results, with a point estimate close to 1 as a strong indicator of no effect. Furthermore, we illustrated that after removing colliders from the model, both logistic regression and the AIPW procedure reported similar results.

Conditioning on collider results in a selection bias that is different from a confounding bias. ${ }^{18}$ Confounding bias arises due to the existence of a common cause of exposure (sex) and outcome (CRT response). Selection bias results from conditioning on a common effect of exposure and outcome. Importantly, we had no time-updated covariate data in this study, and all the covariates were measured at the baseline. Nevertheless, our study illustrates the complexity and challenges of the study of sex exposure, producing time-varying and time-modified confounding and selection bias. We admit that our classification of covariates on confounders and colliders is somewhat arbitrary, semantic and might be study specific. Notably, our study elucidated the limitations of logistic regression analysis for the assessment of sex exposure, producing vastly different estimates, depending on the composition of covariates set.

Doubly robust AIPW procedure is an advanced statistical procedure ${ }^{16}$ that estimates the ATE from observational data by AIPW, which can be viewed as a new generation of propensity score matching. AIPW estimators combine aspects of regression adjustment and inverse-probability-weighted methods. The double-robust property of AIPW estimators permits the misspecification of one out of the two models. It was illustrated by AIPW model 1, which was not balanced, yet reported a consistent point estimate. As soon as the original SMART-AV randomised controlled trial (RCT) did not prespecify the assessment of the heterogeneity of treatment effect by sex, and women comprised only one-third of the study participants, the traditional approach ${ }^{410}$ of measuring unadjusted (or minimally adjusted) interaction by sex in regression analysis is insufficient.

Our study adds to the significant body of evidence indicating that fundamentally, men and women respond to CRT similarly. ${ }^{5-7}$ Men and women have substantial differences in underlying disease substrate, degree and 
Overlaps of densities

of the probabilities of being male (score 0 ) or female (score 1)

in

Augmented-Inverse-Probability-Weighted

(AIPW) models

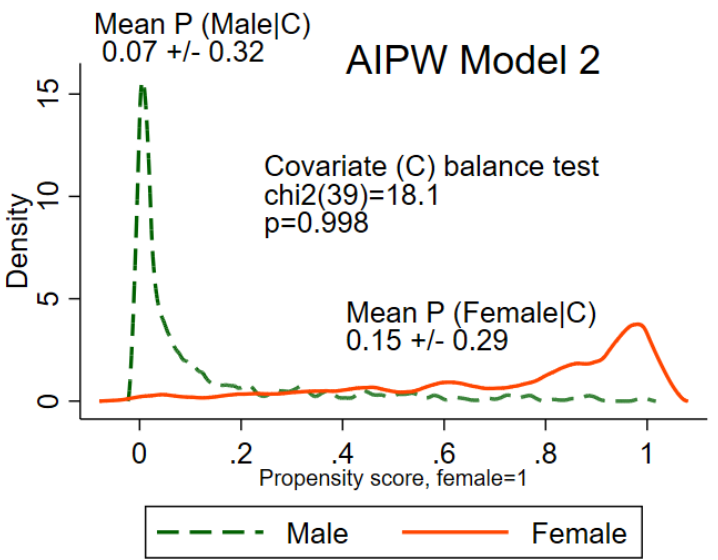

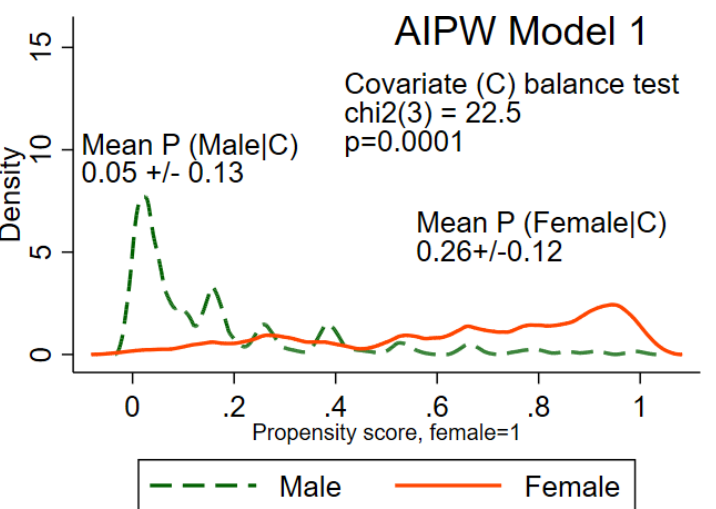

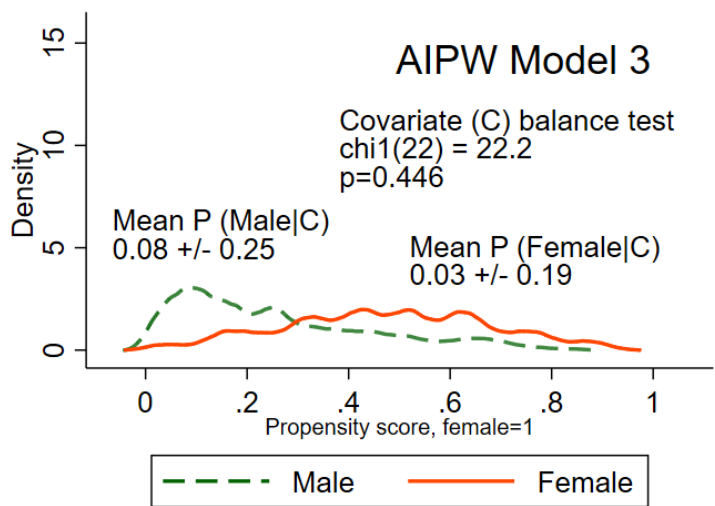

Figure 5 An overlap plot for the estimated densities of the probabilities of being male $(\mathrm{score}=0)$ or female $(\mathrm{score}=1)$, conditioned for covariates included in augmented-inverse-probability-weighted (AIPW) models 1-3.

characteristics of dyssynchrony, ${ }^{5}$ a spectrum of comorbidities and HF treatment. Therefore, many previous studies concluded that CRT is more beneficial for women than men ${ }^{10}$ and described sex differences in CRT response. ${ }^{419}$

Our study used a powerful counterfactual causal inference analytical approach ${ }^{20}$ and demonstrated that both sexes benefit from CRT similarly. Sex-linked biological characteristics can influence health through socially modifiable experiences (eg, poverty, violence, discrimination). Conclusion about the similar benefit for both sexes is important; it demystifies sex-specific CRT response and removes ground for sex inequality. Further studies using a counterfactual framework to study the effect of sex on health outcomes are needed..$^{20}$ Nevertheless, our finding of similar benefits from CRT for both sexes does not negate well-documented sex differences, ${ }^{21}{ }^{22}$ including differences in dyssynchrony, HF substrate and response to pacing therapy ${ }^{10}$ which have to be studied further.

Limitations of the study must be acknowledged. Per the design, the SMART-AV RCT completed a 6-month follow-up, and there was no data on long-term outcomes. Nevertheless, 6-month CRT response is a well-established predictor of long-term survival, ${ }^{23-26}$ and we expect that the sex differences in baseline covariates can also explain the sex differences in long-term outcomes. Furthermore, observed in this study, rate of CRT non-response

Table 2 Sensitivity analysis.

Model

Unadjusted logistic regression

Logistic regression model 1

Logistic regression model 2

Logistic regression model 3

\section{OR $(95 \% \mathrm{Cl})$}

1.95 (1.42 to 2.68$)$

2.32 (1.51 to 3.55$)$

1.79 (1.07 to 2.99$)$

$1.12(0.77$ to 1.62$)$

\section{Average treatment effect $(95 \% \mathrm{Cl})$}

Augmented-inverse-probability-weighted model 1 1.05 (0.60 to 1.86$)$

Augmented-inverse-probability-weighted model 2

0.91 (0.49 to 1.68$)$

Augmented-inverse-probability-weighted model 3 $1.07(0.97$ to 1.18$)$

Relative odds of the volumetric CRT response for women compared with men, by logistic regression and augmented-inverse-probabilityweighted average treatment effect estimator.

CRT, cardiac resynchronisation therapy. 
is higher than widely cited $30 \% .^{2}$ Our definition of CRT response included both volumetric remodelling and clinical outcomes (death, HF hospitalisation) for participants who did not have 6-month post-CRT echocardiographic measurements, which reduced attrition bias and, therefore, strengthened the study. To assess whether CRT response definition affects the relative comparison of CRT response in men and women, we constructed a sensitivity analysis, which provided consistent results.

\section{CONCLUSION}

In summary, in this study, we showed that both sexes' response to CRT is similar, as outcome disparities between sex subgroups are explained by differences in disease substrate, characteristics of dyssynchrony, comorbidities and HF treatment.

\section{Twitter Larisa G Tereshchenko @Tereshchenkolab}

Contributors SH and LGT designed the current study. TMS, KS and KE conducted the original SMART-AV RCT, collected the data and provided the dataset for analysis. SJH drafted the manuscript. LGT conducted statistical analyses, prepared the figures and tables and critically revised the manuscript. All authors interpreted the study findings and revised the manuscript. All authors approved the submitted version and have agreed both to be personally accountable for the author's own contributions and the accuracy and integrity of any part of the work. LGT accepts full responsibility for the work and the conduct of the study, had access to the data, and controlled the decision to publish.

Funding This work was supported in part by the National Heart, Lung, and Blood Institute (HL118277 to Tereshchenko). Boston Scientific Corporation funded the SMART-AV trial.

Competing interests The SMART-AV trial was sponsored by Boston Scientific Corporation. Two co-authors are the Boston Scientific Corporation employees. Boston Scientific had role in the design of this study and had no any role during its execution, analyses, interpretation of the data, or decision to submit results.

Patient consent for publication Consent obtained directly from patient(s)

Ethics approval All SMART-AV participants provided written informed consent before entering the trial. The authors used the deidentified SMART-AV dataset provided by the executive study committee. The Oregon Health \& Science University Institutional Review Board reviewed the current study (OHSU IRB ID STUDY00015297) and determined that due to the dataset's deidentified nature, 'the proposed activity is not research involving human subjects. IRB review and approval is not required'.

Provenance and peer review Not commissioned; externally peer reviewed.

Data availability statement Data may be obtained from a third party and are not publicly available.

Supplemental material This content has been supplied by the author(s). It has not been vetted by BMJ Publishing Group Limited (BMJ) and may not have been peer-reviewed. Any opinions or recommendations discussed are solely those of the author(s) and are not endorsed by BMJ. BMJ disclaims all liability and responsibility arising from any reliance placed on the content. Where the content includes any translated material, BMJ does not warrant the accuracy and reliability of the translations (including but not limited to local regulations, clinical guidelines, terminology, drug names and drug dosages), and is not responsible for any error and/or omissions arising from translation and adaptation or otherwise.

Open access This is an open access article distributed in accordance with the Creative Commons Attribution Non Commercial (CC BY-NC 4.0) license, which permits others to distribute, remix, adapt, build upon this work non-commercially, and license their derivative works on different terms, provided the original work is properly cited, appropriate credit is given, any changes made indicated, and the use is non-commercial. See: http://creativecommons.org/licenses/by-nc/4.0/.

\section{ORCID iDs}

Timothy M Stivland http://orcid.org/0000-0001-8169-1701
Larisa G Tereshchenko http://orcid.org/0000-0002-6976-1313

\section{REFERENCES}

1 Tracy CM, Epstein AE, Darbar D, et al. 2012 ACCF/AHA/HRS focused update of the 2008 guidelines for Device-Based therapy of cardiac rhythm abnormalities: a report of the American College of cardiology Foundation/American heart association Task force on practice guidelines. Heart Rhythm 2012;9:1737-53.

2 Chatterjee NA, Singh JP. Cardiac resynchronization therapy: past, present, and future. Heart Fail Clin 2015;11:287-303.

3 Cheng Y-J, Zhang J, Li W-J, et al. More favorable response to cardiac resynchronization therapy in women than in men. Circ Arrhythm Electrophysiol 2014;7:807-15.

4 Arshad A, Moss AJ, Foster E, et al. Cardiac resynchronization therapy is more effective in women than in men: the MADIT-CRT (multicenter automatic defibrillator implantation trial with cardiac resynchronization therapy) trial. J Am Coll Cardiol 2011;57:813-20.

5 Beela AS, Duchenne J, Petrescu A, et al. Sex-Specific difference in outcome after cardiac resynchronization therapy. Eur Heart $J$ Cardiovasc Imaging 2019;20:504-11.

6 Linde C, Abraham WT, Gold MR, et al. Randomized trial of cardiac resynchronization in mildly symptomatic heart failure patients and in asymptomatic patients with left ventricular dysfunction and previous heart failure symptoms. J Am Coll Cardiol 2008;52:1834-43.

7 Bristow MR, Saxon LA, Boehmer J, et al. Cardiac-Resynchronization therapy with or without an implantable defibrillator in advanced chronic heart failure. N Engl J Med 2004;350:2140-50.

8 Singh JP, Fan D, Heist EK, et al. Left ventricular lead electrical delay predicts response to cardiac resynchronization therapy. Heart Rhythm 2006;3:1285-92.

9 Gold MR, Yu Y, Singh JP, et al. Effect of interventricular electrical delay on atrioventricular optimization for cardiac resynchronization therapy. Circ Arrhythm Electrophysiol 2018;11:e006055.

10 Cheng A, Gold MR, Waggoner AD, et al. Potential mechanisms underlying the effect of gender on response to cardiac resynchronization therapy: insights from the SMART-AV multicenter trial. Heart Rhythm 2012;9:736-41.

11 Stein KM, Ellenbogen KA, Gold MR, et al. SmartDelay determined AV optimization: a comparison of AV delay methods used in cardiac resynchronization therapy (SMART-AV): rationale and design. Pacing Clin Electrophysiol 2010;33:54-63.

12 Ellenbogen KA, Gold MR, Meyer TE, et al. Primary results from the SmartDelay determined AV optimization: a comparison to other AV delay methods used in cardiac resynchronization therapy (SMARTAV) trial: a randomized trial comparing empirical, echocardiographyguided, and algorithmic atrioventricular delay programming in cardiac resynchronization therapy. Circulation 2010;122:2660-8.

13 Matsushita K, Selvin E, Bash LD, et al. Risk implications of the new CKD epidemiology collaboration (CKD-EPI) equation compared with the MDRD study equation for estimated GFR: the Atherosclerosis risk in communities (ARIC) study. Am J Kidney Dis 2010;55:648-59.

14 Field ME, Yu N, Wold N, et al. Comparison of measures of ventricular delay on cardiac resynchronization therapy response. Heart Rhythm 2020;17:615-20.

15 Tereshchenko LG, Cheng A, Park J, et al. Novel measure of electrical dyssynchrony predicts response in cardiac resynchronization therapy: results from the SMART-AV trial. Heart Rhythm 2015;12:2402-10.

16 Cattaneo MD. Efficient semiparametric estimation of multi-valued treatment effects under ignorability. J Econom 2010;155:138-54.

17 Imai K, Ratkovic M. Covariate balancing propensity score. J. R. Stat. Soc. B 2014;76:243-63.

18 Cole SR, Platt RW, Schisterman EF, et al. Illustrating bias due to conditioning on a collider. Int J Epidemiol 2010;39:417-20.

19 Jamerson D, McNitt S, Polonsky S, et al. Early procedure-related adverse events by gender in MADIT-CRT. $J$ Cardiovasc Electrophysiol 2014;25:985-9.

20 Glymour MM, Spiegelman D. Evaluating public health interventions: 5. causal inference in public health Research-Do sex, race, and biological factors cause health outcomes? Am J Public Health 2017;107:81-5.

21 Howell SJ, German D, Bender A, et al. Does sex modify an association of electrophysiological substrate with sudden cardiac death? The Atherosclerosis risk in communities (ARIC) study. Cardiovasc Digit Health J 2020;1:80-8.

22 Wang L, Javadekar N, Rajagopalan A, et al. Eligibility for subcutaneous implantable cardioverter-defibrillator in congenital heart disease. Heart Rhythm 2020;17:860-9. 
23 White HD, Norris RM, Brown MA, et al. Left ventricular end-systolic volume as the major determinant of survival after recovery from myocardial infarction. Circulation 1987;76:44-51.

24 Yu C-M, Bleeker GB, Fung JW-H, et al. Left ventricular reverse remodeling but not clinical improvement predicts long-term survival after cardiac resynchronization therapy. Circulation 2005;112:1580-6.
25 Bertini M, Höke U, van Bommel RJ, et al. Impact of clinical and echocardiographic response to cardiac resynchronization therapy on long-term survival. Eur Heart J Cardiovasc Imaging 2013;14:774-81.

26 Stankovic I, Belmans A, Prinz C, et al. The association of volumetric response and long-term survival after cardiac resynchronization therapy. Eur Heart J Cardiovasc Imaging 2017;18:1109-17. 